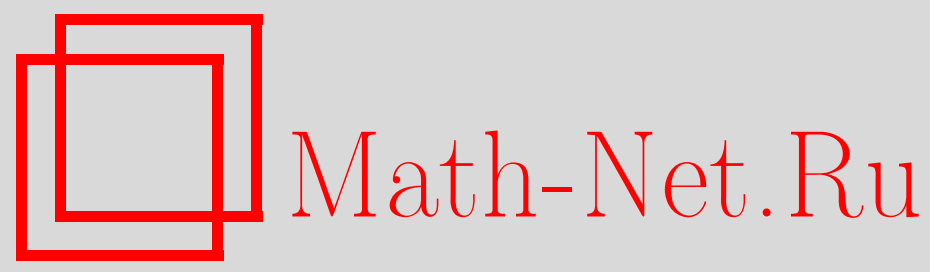

Я. Б. Воробец, А. М. Степин, Изоспектральность и проективные геометрии Галуа, Матем. заметки, 1998, том 63, выпуск 5, 660-664

DOI: https://doi.org/10.4213/mzm1331

Использование Общероссийского математического портала Math-Net.Ru подразумевает, что вы прочитали и согласны с пользовательским соглашением http://www . mathnet.ru/rus/agreement

Параметры загрузки:

IP : 54.80 .97 .219

26 апреля 2023 г., 13:26:04

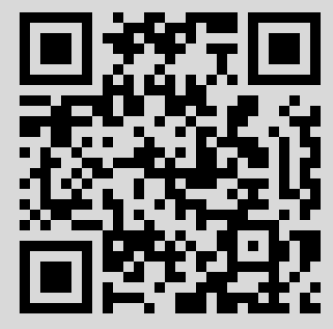




\title{
ИЗОСПЕКТРАЛЬНОСТЬ И ПРОЕКТИВНЫЕ ГЕОМЕТРИИ ГАЛУА
}

\author{
Я.Б. Воробец, А. М. Стёпин
}

\begin{abstract}
Предложена конструкция, приводящая к серии пар изоспектральных и неизометричных плоских областей и поверхностей с краем. Эта конструкция основана на существовании спектрально эквивалентных, но не изоморфных, конечных групп преобразований.

Библиограффия: 4 названия.
\end{abstract}

В известной работе 1966 г. М. Кац поставил следующий вопрос: влечет ли изоспектральность областей в $\mathbb{R}^{2}$ (т.е. совпадение спектров соответствующих операторов Лапласа) их изометричность. Л. Берс перефразировал вопрос популярно: можно ли услышать форму барабана? В 1992 г. К. Гордон, Д. Уэбб и С. Вольперт [1] дали отрицательный ответ на этот вопрос.

В настоящей работе указано на связь проблемы изоспектральности с конечными проективньми геометриями и предложена конструкция, включающая пример из [1] в серию изоспектральных неизометричных областей (и многообразий с краем). Наша конструкция базируется на существовании спектрально эквивалентных, но несопряженных групп преобразований. Такие группы впервые рассматривались в алгебраической теории чисел. Связь этих групп с изоспектральными и неизометричными многообразиями впервые подметил Т. Сунада [2]. Доказательство изоспектральности в [2] было проинтерпретировано П. Бераром [3] как процедура трансплантации функций; это привело в [3] к обобщению конструкции Сунады на орбиобразия ( orbifolds). Последнее позволило авторам упомянутой работы [1] сделать переход от многообразий к областям в $\mathbb{R}^{2}$.

Изложение начнем с простейшего, на наш взгляд, примера изоспектральных, но неконгруэнтных, областей на плоскости. Пусть $T$ - остроугольньй треугольник. Из четырнадцати экземпляров этого треугольника составим фигуры $F^{(1)}$ и $F^{(2)}$ так, как показано на рис. $1,2$.

ПРЕДЛОЖЕНИЕ 1. а) Операторы Лапласа в областях $F^{(1)}$ u $F^{(2)}$ имеют одинаковый спектр как при краевых условиях Дирихле, так и при краевых условиях Неймана.

б) Если все углы треугольника $T$ различны, то многоугольники $F^{(1)}$ и $F^{(2)}$ неконгруәнтны.

Теперь опишем общую конструкцию, частным случаем которой является рассмотренный только что пример.

Работа вьполнена при частичной поддержке объединенного проекта фонда INTAS и Российского фонда фундаментальных исследований, грант № 96-01-00043. 


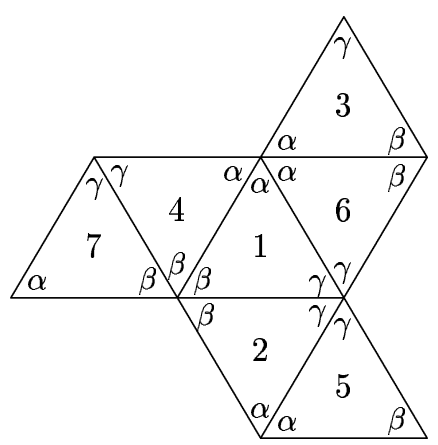

Рис. 1. Многоугольник $F^{(1)}$

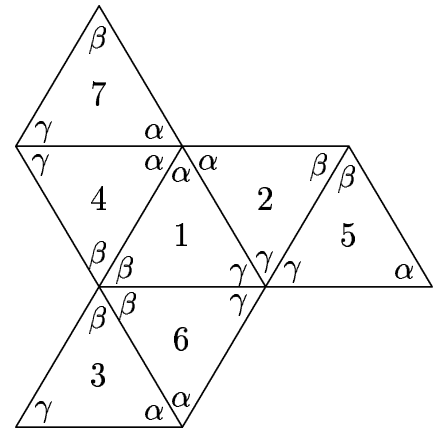

Рис. 2. Многоугольник $F^{(2)}$

Пусть $G$ - конечная группа, $g_{1}, \ldots, g_{k}$ - ее образующие. Базовой фигурой, соответствующей этой системе образующих, назовем произвольную плоскую фигуру $T$, у которой имеется несколько прямолинейных сегментов границы, помеченных элементами $g_{1}, \ldots, g_{k}$ и их обратными (если элемент $g_{i}$ имеет порядок 2 , то метки $g_{i}$ и $g_{i}^{-1}$ совпадают и относятся к одному и тому же сегменту). Указанные сегменты назовем сторонами фигуры T. Предполагается, что

i) стороны, помеченные разными элементами групшы, не перекрываются (могут иметь лиш общие конщы);

ii) стороны, помеченные взаимно обратными элементами $\left(g_{i}\right.$ и $\left.g_{i}^{-1}\right)$, имеют одинаковую длину;

iii) все стороны каким-то образом ориентированы (независимо друг от друга).

Базовую фигуру можно представлять в виде многоугольника со срезанными углами. Объединение всех срезов, т.е. ту часть границы фигуры $T$, которая остается после выбрасьвания всех сторон, назовем свободной границей.

Пусть, далее, $\varphi=\left\{\varphi_{g}\right\}_{g \in G}$ - действие грушы $G$ на множестве $M$. Составной физурой, соответствующей действию $\varphi$ и базовой фигуре $T$, назьвается фигура $F$, составленная из $n=\# M$ фигур $T_{m}, m \in M$, таким образом, что

i) каждая из фигур $T_{m}$ изометрична базовой фигуре $T$, с учетом меток и ориентации сторон;

ii) разные фигуры не перекрываются, но могут иметь общие стороны, причем эти общие стороны ориентированы согласованно;

iii) если $g \in\left\{g_{1}, \ldots, g_{k}, g_{1}^{-1}, \ldots, g_{k}^{-1}\right\}$, то сторона $T_{m}$ с меткой $g$ совпадает со стороной фигуры $T_{\varphi_{g}(m)}$, имеющей метку $g^{-1}$.

В дальнейшем рассматриваются только транзитивные действия $\varphi$; соответствующие им составные фигуры являются связными.

Пусть, наконец, $\varphi^{(1)}$ и $\varphi^{(2)}-$ действия групшы $G$ на $n$-элементных множествах $M^{(1)}$ и $M^{(2)}$. Выберем произвольньм образом базовую фигуру $T$ и построим соответствуюшие составные фигуры $F^{(1)}$ и $F^{(2)}$. Оказьвается (и об этом говорится далее), что в случае спектральной эквивалентности действий $\varphi^{(1)}$ и $\varphi^{(2)}$ поверхности $\left(\right.$ к краем $F^{(1)}$ и $F^{(2)}$ изоспектральны. Если $\varphi^{(1)}$ и $\varphi^{(2)}$ не сопряжены, то для типичной базовой фигуры $T$ поверхности $F^{(1)}$ и $F^{(2)}$ неизометричны.

Пусть $A=\left(a\left(j_{2}, j_{1}\right)\right)$ - произвольная матрица размера $n \times n$, строки которой индексированы элементами множества $M^{(2)}$, а столбцы - элементами множества $M^{(1)}$. Сопоставим этой матрице оператор трансплантации $U_{A}: L_{2}\left(F^{(1)}\right) \rightarrow L_{2}\left(F^{(2)}\right)$ следуюшим образом.

Произвольная функция $f^{(i)}$ на $F^{(i)}$ может быть представлена как набор из $n$ функций 
$f_{j}^{(i)}, j \in M^{(i)}$, заданных на $T$, где функция $f_{j}^{(i)}$ отождествляется с ограничением функции $f^{(i)}$ на фигуру $T_{j}^{(i)}$. Зададим оператор $U_{A}: f^{(1)}=\left(f_{j}^{(1)}\right)_{j \in M^{(1)}} \mapsto f^{(2)}=\left(f_{j}^{(2)}\right)_{j \in M^{(2)}}$ формулой

$$
f_{j_{2}}^{(2)}=\sum_{j_{1} \in M^{(1)}} a\left(j_{2}, j_{1}\right) f_{j_{1}}^{(1)} .
$$

Кроме того, рассмотрим оператор $u_{A}: \ell_{2}\left(M^{(1)}\right) \rightarrow \ell_{2}\left(M^{(2)}\right)$, задаваемый матрицей $A$ в базисе из $\delta$-функций $\chi_{j}^{(i)}$ (т.е. индикаторов одноточечных множеств).

Прежде чем сформулировать условие, при котором $U_{A}$ сплетает операторы Лапласа на $F^{(1)}$ и $F^{(2)}$, введем одно ограничение на геометрию составных фигур. Скажем, что фигура $F^{(i)}$ удовлетворяет условию $(*)$, если угловые точки (т.е. общие концы двух сторон) составляющих ее фигур $T_{j}^{(i)}$ не являются внутренними точками $F^{(i)}$. Ecли условие $(*)$ не вьполнено, можно добиться его выполнения путем изменения базовой фигуры - нужно срезать у последней один или несколько углов.

ПРЕДЛОЖЕНИЕ 2. Пусть фигуры $F^{(1)}$ и $F^{(2)}$ удовлетворяют условию (*). Oператор трансплантачии $U_{A}$ сплетает операторы Лапласа с краевыми условиями Неймана в том и только том случае, когда оператор и сплетает естественные представления $G$ в $\ell_{2}\left(M^{(1)}\right)$ и $\ell_{2}\left(M^{(2)}\right)$. В частности, спектральная әквивалентность действий $\varphi^{(1)}$ и $\varphi^{(2)}$ влечет совпадение спектров операторов Лапласа в $F^{(1)} u F^{(2)}$.

Имеется аналогичное утверждение и об операторах Лапласа с краевыми условиями Дирихле.

Кроме изоспектральности, от фигур $F^{(1)}$ и $F^{(2)}$ нам требуется также и неизометричность. Об этом нужно специально позаботиться, так как даже для несопряженных действий $\varphi^{(1)}$ и $\varphi^{(2)}$ при неудачном выборе базовой фигуры может оказаться, что составные фигуры изометричны. Сформулируем здесь следующее

ПРЕДЛОЖЕНИЕ 3. ДЛя любой конечной группы $G$ с выделенными образующими можно в качестве базовой фигуры выбрать такую плоскую область с кусочно-гладкой границей, что

i) составная фигура, построенная по этой базовой фигуре и произвольному действию группы $G$, удовлетворяет условию (*);

ii) составные фигуры, построенные по несопряжсенным действиям, неизометричны.

Таким образом, задача поиска изоспектральных и неизометричных областей сводится к нахождению конечных груп, обладающих парой спектрально эквивалентных и несопряженных действий. Основным источником таких действий для нас будут конечные проективные геометрии (точнее, геометрии Галуа).

Рассмотрим произвольную геометрию Галуа, т.е. конечномерное проективное пространство $M^{(1)}$ над конечньм полем. Пусть $M^{(2)}$ - множество ее гиперплоскостей (прямых в случае проективной плоскости). Обозначим через $G$ группу проективных преобразований рассматриваемой геометрии. Пусть $\varphi^{(1)}$ и $\varphi^{(2)}-$ естественные действия группы $G$ на множествах $M^{(1)}$ и $M^{(2)}$ соответственно.

ПРЕДЛОЖЕНИЕ 4. Действия $\varphi^{(1)}$ и $\varphi^{(2)}$ спектрально әквивалентны, но не сопрязсены.

Итак, теперь остается выбрать образующие групшы $G$, и можно получать примеры 
изоспектральных и неизометричных плоских поверхностей с краем (в частности, областей в $\mathbb{R}^{2}$ ). Отметим, что образуюшие можно выбирать многими способами и это приводит к различным примерам.

Теорема 1. Существует бесконечная серия пар изоспектральных и неизометричных двумерных многообразий с краем, имеющих плоскую метрику.

Это утверждение требует пояснения. Очевидно, достаточно лишь одной пары спектрально эквивалентных и несопряженных действий конечной групшы, чтобы получить семейство пар изоспектральных и неизометричных поверхностей, которые получаются друг из друга деформацией базовой фигуры. Теорема утверждает существование бесконечного числа пар, ни одна из которых не может быть сведена к другой посредством такой деформации.

Теперь опишем ряд модификаций изложенной вьше конструкции изоспектральных областей и поверхностей, которые позволяют обобщить теорему 1 в различных направлениях. Во-первых, можно перейти от двумерньх фигур к многомерньм. Далее, евклидову метрику можно заменить на произвольную риманову метрику. В качестве базовой фигуры теперь может выступать конечномерное риманово многообразие с краем, а роль сторон или граней играют неперекрывающиеся гладкие куски гранищы базовой фигуры. Сборка составного многообразия с краем из отдельных копий базовой фигуры производится посредством отображений $\psi_{1}, \ldots, \psi_{k}$, где $\psi_{i}-$ изометрия грани базовой фигуры с меткой $g_{i}$ на грань, имеющую метку $g_{i}^{-1}$. Гладкость метрики на составной фигуре обеспечивается дополнительным условием на базовую фигуру. Еще одна модификация связана с пересмотром краевых условий. Если изменить их согласованно на свободной границе, то предложение 2 будет по-прежнему верно. В результате оператор трансплантации, сплетаюший операторы Лапласа с краевыми условиями Неймана, заданные на составных многообразиях с непустой свободной границей, сплетает также и целое семейство пар операторов Лапласа на этих многообразиях со смешанными (частично Неймана, частично Дирихле) краевыми условиями.

Наконец, последнее усовершенствование относится к кратности изоспектральнос$m u$, т.е. к вопросу о возможном числе попарно неизометричных изоспектральных многообразий. Применяя нашу основную конструкцию (в модифицированном виде) к всевозможным тензорным произведениям спектрально эквивалентных, но не сопряженных действий групшы $G$, получим набор попарно неизометричных изоспектральных областей или многообразий с краем.

Один из способов подытожить эти наблюдения таков.

ТЕорема 2. Для любых иелых чисел $l, n \geqslant 2$ и любого действительного числа $R$ существует бесконечная серия наборов из $l$ изоспектральных и попарно неизометричных п-мерных многообразий с краем, с римановой метрикой постоянной кривизны $R$.

Теперь приведем ряд найденных нами примеров спектрально эквивалентных и несопряженных действий. В каждом из них действует группа проективных преобразований конечной геометрии, имеющая три инволютивные образующие $a, b, c$. Перестановки $a_{1}, b_{1}, c_{1}$ задают действие этих образующих на точках проективной геометрии, а перестановки $a_{2}, b_{2}, c_{2}$ - их действие на прямых (соответственно на плоскостях в случае геометрии порядка 15). Пример 1) был рассмотрен в предложении 1, пример 2) был найден в [1].

1) $a_{1}=(1,2)(4,7), b_{1}=(1,6)(2,5), c_{1}=(1,4)(3,6) ; a_{2}=b_{1}, b_{2}=a_{1}, c_{2}=c_{1}$;

2) $a_{1}=(1,5)(3,7), b_{1}=(1,2)(5,6), c_{1}=(1,4)(3,6) ; a_{2}=(4,5)(6,7), b_{2}=b_{1}$, $c_{2}=c_{1}$ 
3) $a_{1}=(2,6)(3,7), b_{1}=(1,2)(5,6), c_{1}=(1,4)(3,6) ; a_{2}=(4,6)(5,7), b_{2}=b_{1}$, $c_{2}=c_{1}$

4) $a_{1}=(1,8)(2,6)(3,13)(7,11), b_{1}=(1,5)(3,8)(4,11)(10,13), c_{1}=(6,7)(8,11)(9,13)$ $(10,12) ; a_{2}=(1,2)(5,13)(6,10)(8,12), b_{2}=b_{1}, c_{2}=c_{1}$

5) $a_{1}=(1,8)(2,7)(4,12)(6,11), b_{1}=(1,5)(3,8)(4,11)(10,13), c_{1}=(6,7)(8,11)(9,13)$ $(10,12) ; a_{2}=(1,2)(5,12)(7,9)(8,13), b_{2}=b_{1}, c_{2}=c_{1}$;

6) $a_{1}=(1,8)(2,13)(3,6)(5,12), b_{1}=(1,5)(3,8)(4,11)(10,13), c_{1}=(6,7)(8,11)(9,13)$ $(10,12) ; a_{2}=(1,8)(2,12)(4,7)(5,13), b_{2}=b_{1}, c_{2}=c_{1}$

7) $a_{1}=(1,9)(2,5)(4,13)(7,12), b_{1}=(1,5)(3,8)(4,11)(10,13), c_{1}=(6,7)(8,11)(9,13)$ $(10,12) ; a_{2}=(2,7)(3,6)(4,5)(8,9), b_{2}=b_{1}, c_{2}=c_{1}$

8) $a_{1}=(1,13)(2,8)(3,6)(7,9), b_{1}=(1,5)(3,8)(4,11)(10,13), c_{1}=(6,7)(8,11)(9,13)$ $(10,12) ; a_{2}=(2,4)(5,13)(6,11)(7,12), b_{2}=b_{1}, c_{2}=c_{1}$

9) $a_{1}=(3,4)(5,8)(6,9)(7,10), b_{1}=(1,8)(2,6)(3,13)(7,11), c_{1}=(6,7)(8,11)(9,13)$ $(10,12) ; a_{2}=(2,11)(3,12)(4,13)(9,10), b_{2}=(1,2)(5,13)(6,10)(8,12), c_{2}=c_{1}$;

10) $a_{1}=(3,4)(5,11)(6,12)(7,13), b_{1}=(1,8)(2,6)(3,13)(7,11), c_{1}=(6,7)(8,11)(9,13)$ $(10,12) ; a_{2}=(2,8)(3,10)(4,9)(12,13), b_{2}=(1,2)(5,13)(6,10)(8,12), c_{2}=c_{1}$

11) $a_{1}=(1,9)(3,12)(4,6)(5,11), b_{1}=(1,8)(2,6)(3,13)(7,11), c_{1}=(6,7)(8,11)(9,13)$ $(10,12) ; a_{2}=(2,9)(3,10)(4,8)(5,7), b_{2}=(1,2)(5,13)(6,10)(8,12), c_{2}=c_{1}$;

12) $a_{1}=(1,13)(3,10)(4,7)(5,8), b_{1}=(1,8)(2,6)(3,13)(7,11), c_{1}=(6,7)(8,11)(9,13)$ $(10,12) ; a_{2}=(2,13)(3,12)(4,11)(5,6), b_{2}=(1,2)(5,13)(6,10)(8,12), c_{2}=c_{1}$

13) $a_{1}=(1,10)(3,8)(4,15)(6,13), b_{1}=(1,4)(3,6)(9,12)(11,14), c_{1}=(1,8)(2,4)(3,12)$ $(5,10)(7,14)(11,13) ; a_{2}=(1,12)(2,15)(5,8)(6,11), b_{2}=b_{1}, c_{2}=c_{1}$

14) $a_{1}=(1,6)(2,5)(8,15)(11,12), b_{1}=(1,4)(3,6)(9,12)(11,14), c_{1}=(1,8)(2,4)(3,12)$ $(5,10)(7,14)(11,13) ; a_{2}=(1,10)(2,9)(4,15)(7,12), b_{2}=b_{1}, c_{2}=c_{1}$;

15) $a_{1}=(1,2)(5,6)(8,11)(12,15), b_{1}=(1,12)(3,14)(4,9)(6,11), c_{1}=(1,8)(2,4)(3,12)$ $(5,10)(7,14)(11,13) ; a_{2}=(1,10)(2,9)(5,14)(6,13), b_{2}=(1,4)(3,6)(8,13)(10,15)$, $c_{2}=c_{1}$

16) $a_{1}=(1,13)(3,15)(4,8)(6,10), b_{1}=(1,12)(3,14)(4,9)(6,11), c_{1}=(1,8)(2,4)(3,12)$ $(5,10)(7,14)(11,13) ; a_{2}=(4,9)(5,8)(6,11)(7,10), b_{2}=(1,4)(3,6)(8,13)(10,15)$, $c_{2}=c_{1}$.

Каждьй из примеров 1)-16) позволяет получить пару многоугольников на плоскости Евклида или Лобачевского, которые изоспектральны, но не изометричны. Более того, если в качестве базовой фигуры взять треугольник на плоскости Лобачевского с достаточно малыми углами, можно добиться, чтобы эти многоугольники были выпуклыми (для примера 2) на это указали Гордон и Уэбб [4]).

Основная для наших целей - связь изоспектральности с конечньми геометриями - была обнаружена авторами весной 1996 г. и доложена в январе 1997 г. на семинаре К. Шмидта в институте Шрёдингера.

\section{СПИСОК ЦИТИРОВАННОЙ ЛИТЕРАТУРЫ}

[1] Gordon C., Webb D., Wolpert S. Isospectral plane domains and surfaces via Riemannian orbifolds // Invent. Math. 1992. V. 110. № 1. P. 1-22.

[2] Sunada T. Riemannian coverings and isospectral manifolds // Ann. of Math. (2). 1985. V. 121. P. $169-186$.

[3] Berard P. Transplantation et isospéctralité. I // Math. Ann. 1992. V. 292. № 3. P. 547-560.

[4] Gordon C. S., Webb D. L. Isospectral convex domains in the hyperbolic plane // Proc. Amer. Math. Soc. 1994. V. 120. № 3. P. 981-983. 